\title{
A flexible and fast PyTorch toolkit for simulating training and inference on analog crossbar arrays*
}

\author{
Malte J. Rasch ${ }^{1}$, Diego Moreda ${ }^{1}$, Tayfun Gokmen ${ }^{1}$, Manuel Le \\ Gallo $^{1}$, Fabio Carta ${ }^{1}$, Cindy Goldberg ${ }^{1}$, Kaoutar El Maghraoui ${ }^{1}$, \\ Abu Sebastian ${ }^{1}$, and Vijay Narayanan ${ }^{1}$ \\ ${ }^{1}$ IBM Research
}

April 7, 2021

\begin{abstract}
We introduce the IBM Analog Hardware Acceleration Kit, a new and first of a kind open source toolkit to simulate analog crossbar arrays in a convenient fashion from within PYTORCH (freely available at https://github.com/IBM/aihwkit). The toolkit is under active development and is centered around the concept of an "analog tile" which captures the computations performed on a crossbar array. Analog tiles are building blocks that can be used to extend existing network modules with analog components and compose arbitrary artificial neural networks (ANNs) using the flexibility of the PyTorch framework. Analog tiles can be conveniently configured to emulate a plethora of different analog hardware characteristics and their non-idealities, such as device-to-device and cycle-to-cycle variations, resistive device response curves, and weight and output noise. Additionally, the toolkit makes it possible to design custom unit cell configurations and to use advanced analog optimization algorithms such as Tiki-Taka. Moreover, the backward and update behavior can be set to "ideal" to enable hardware-aware training features for chips that target inference acceleration only. To evaluate the inference accuracy of such chips over time, we provide statistical programming noise and drift models calibrated on phase-change memory hardware. Our new toolkit is fully GPU accelerated and can be used to conveniently estimate the impact of material properties and non-idealities of future analog technology on the accuracy for arbitrary ANNs.
\end{abstract}

*submitted to AICAS 2021 


\section{Introduction}

To cope with the ever increasing demand for computing resources in artificial intelligence applications, dedicated hardware accelerators for artificial neural network (ANN) training and inference have been proposed recently. One promising future technology is the use of memristive crossbar arrays for accelerating the ubiquitous matrix-vector multiply and rank-update operations in ANNs by employing in-memory computation of matrices stored as analog quantities in tunable resistive elements [1,7,8,13]. While considerable run-time and performance gain over today's technology is projected for analog hardware in principle [5], noisy and non-linear device characteristics as well as design restrictions such as stationary weight matrices, demand for a new breed of ANN topologies and algorithmic advances adapted to analog technology to unleash its full potential. An ideal outcome of such "analog AI" algorithmic endeavour would be reminiscent of the increase in popularity of convolutional networks once it became clear that their compute is ideally suited to be performed on graphic processing units (GPUs).

To be viable, these new analog algorithms and optimized network architectures need to be tested in a simulator that takes analog hardware constraints and realistic material properties into account. Moreover, these simulations need to be applied on realistically sized deep neural networks (DNNs) and datasets to make informed decisions about hardware design and the expected accuracy.

While there exists a number of current software packages that are dedicated to simulating AI workloads on non-volatile memory elements, notably MLP-NeuroSim [2] and RxNN [9], they are usually not tied to modern AI frameworks, such as PyToRch [11. Such frameworks have become indispensable tools for AI researchers to build new ANN architectures and implement AI workloads. For instance, while MLP-NEURoSim [2] has the ability to handle a great number of realistic hardware models, it is built around a small (by today's standard) MNIST network example, hence lacking the tools to flexibly implement new ANN topologies or simulate larger DNNs. RxNN [9] is based on the rather outdated Caffe framework and only caters to analog chips dedicated to inference, lacking more advanced algorithms and pulse update schemes that are needed for training-enabled chip designs. A recent PyTORCH re-implementation of a subset of the MLP-NEUROSim package using PYTorCH, called DNN+NeuroSim [12, is closest to our framework. However, it does not support fast custom CUDA kernels for in-situ parallel analog update, and also lacks many of the aspects of modern code development, such as modularity, object oriented programming, proper Python binding of fast $\mathrm{C}++$ elements, unit testing, easy Python package installation and so on. To address these limitations, we decided to open source IBM's internal analog AI simulation core with a convenient PYTORCH interface, hence providing adequate software tools for the advancement of algorithmic analog AI. 

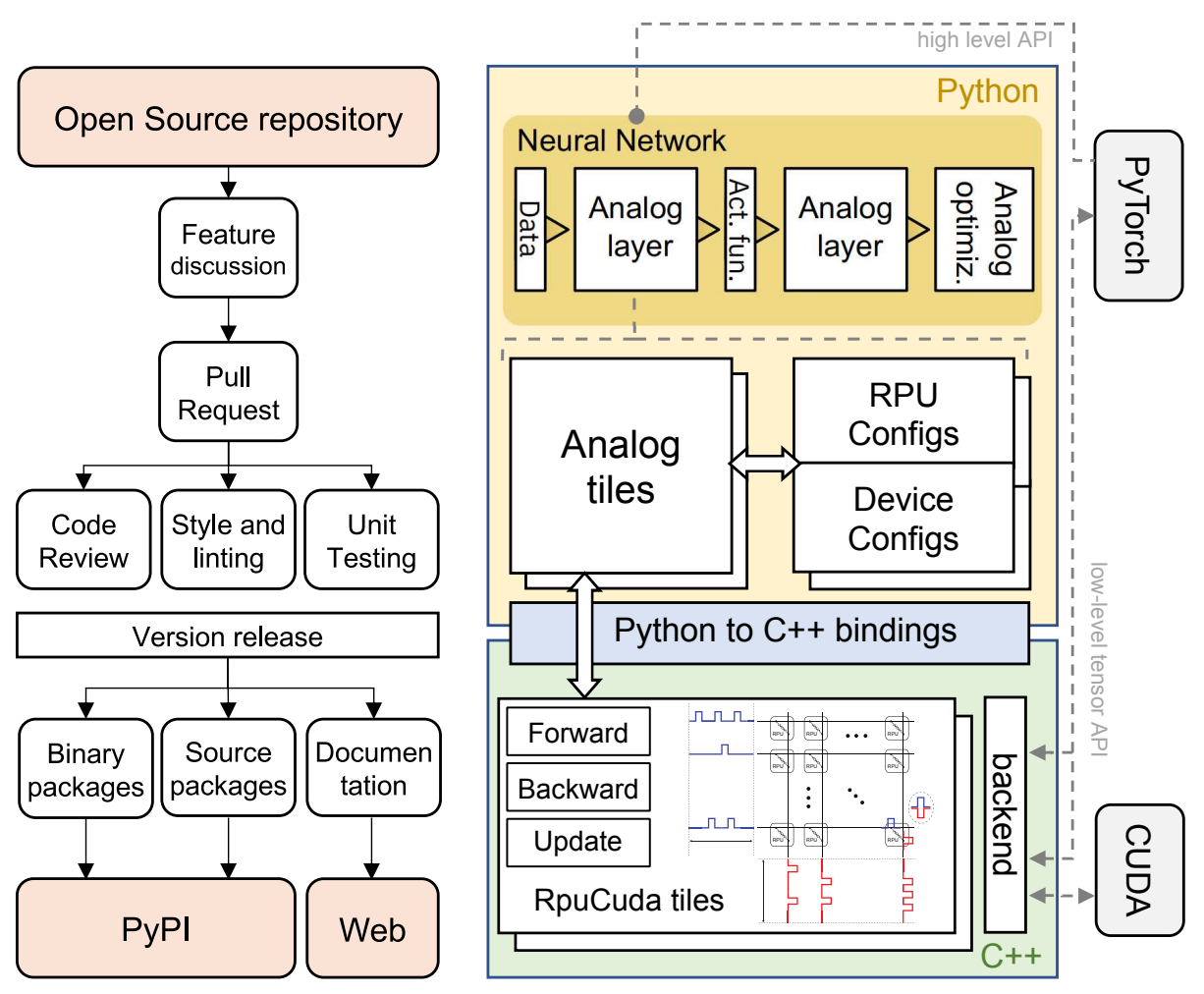

Figure 1: Repository and code structure of the IBM Analog Hardware Acceleration Kit. 


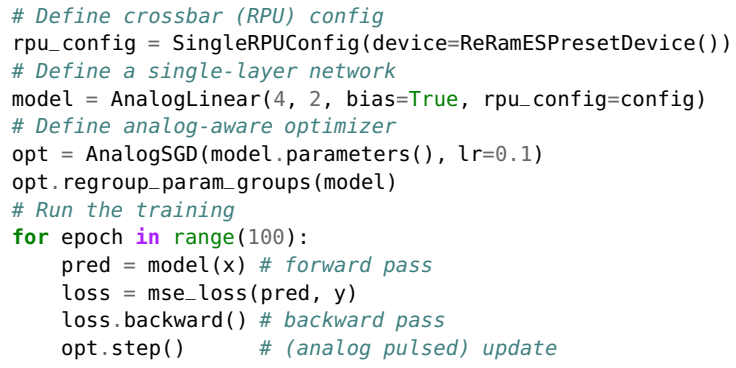

Figure 2: Simple network definition and training with parallel pulsed update. Note that imports and data preparation are omitted for brevity (see example 01 in our GitHub repository).

\section{Toolkit overview}

We provide a professionally maintained software kit that is easy to install (as simple as pip install aihwkit), has an extensive documentation 1 with a growing number of examples and tutorials, and has a well integrated PYTORCH experience providing a number of new Analog layers, such as linear (fully-connected) layer and convolutions (see Fig. (1). Under the hood, these layers perform the analog hardware simulations using a highly optimized $\mathrm{C}++$ /CUDA core library (RPUCUDA) that is part of the package and made available under an Apache-2.0 Open Source license. The core library is bound to the Python layer so that no re-compilation is needed for defining models and parameters. Instead, all $\mathrm{C}++$ functions and analog hardware defining parameters are conveniently accessible from Python. Figure 1 illustrates the architecture and code structure of the toolkit.

Among others, we provide an AnalogLinear layer, which uses a single ana$\log$ tile to compute the forward pass of a fully-connected layer within a DNN. Defining a simple analog training simulations (with default device parameters) is similar to how PYTORCH to define DNN layers (see example in Fig. 22).

\section{Analog tile model simulation}

The toolkit is centered around an "analog tile" that corresponds to a 2D weight matrix that is stored on a non-volatile resistive crossbar array and includes most pre- and post-processing (such as dynamic input scaling and analog-digital converters) that are based on (and extends) the resistive processing unit (RPU) definitions in [5]. The basic operation of this tile is a matrix-vector product $y_{i}=\sum_{j} w_{i j} x_{j}$, where the matrix elements $w_{i j}$ are assumed to be stored in

1https://aihwkit.readthedocs.io/en/latest/ 
the crossbar (optionally together with the biases). The analog matrix-vector product is, however, corrupted by noise and non-idealities that are defined by the user through parameter configurations of the tile during creation (called rpu_config).

For instance, input/output/weight noise processes, analog-to-digital conversion resolutions, or other non-idealities can be added so that the effective computation simulated is e.g 2 given by

$$
y_{i}=f_{\mathrm{adc}}\left(\sum_{j}\left(w_{i j}+\sigma_{\mathrm{w}} \xi_{i j}\right)\left(f_{\mathrm{dac}}\left(x_{j}\right)+\sigma_{\mathrm{inp}} \xi_{j}\right)+\sigma_{\mathrm{out}} \xi_{i}\right)
$$

where $f_{\text {adc }}$ and $f_{\text {dac }}$ are discretization functions (together with e.g. dynamic scaling and clipping) of the digital-analog conversion process (as given by the hardware design), and $\xi$ are Gaussian noise processes. In our tool, we generally opt for a more abstract functional representation of the underlying hardware (i.e. largely working with normalized units), since in this manner the cause of accuracy loss of different hardware designs become more apparent and specifications can be derived based on parameters relevant to the DNN.

The RPU $\mathrm{C}++$ library is the core of the high-performing implementation of Eq. (1), which extensively uses dedicated and fused GPU kernels. It provides the above basic model, however, internal pre- and post processing (e.g. analog to digital discretization) can be simply turned off or adapted by the user using PYTORCH's functionality.

Within a DNN that is considered to be accelerated with analog crossbar arrays, we generally assume that digital operations can be separated from ana$\log$ operations. For instance, by default, activation functions are performed with digital computing using PYTORCH's library of floating point functions and assuming that analog result values of a matrix vector product are digitized. Moreover, we currently support only parallel read-out schemes and linear analog-digital conversion. However, iterative read-out schemes and shift-andadd for more complicated analog-digital converters could be straightforwardly added in a similar manner using PyTORCH.

While the forward pass includes cycle-to-cycle variations of the resistive materials and circuit noise sources (see Eq.11), for chips dedicated to ANN training, backward and (potentially) update passes need to be similarly simulated to be performed on analog as well. Thus, in our framework, backward pass (the transposed version of the forward pass) can be configured to contain noise and non-linearity similar to the forward pass. In general, these parameters do not need to be the same, to reflect hardware designs that differ in the noisiness in both directions.

A particular strength of our new software framework is the highly-optimized pulse update with tune-able material pulse response properties for chips that are dedicated to perform ANN training. For that, we consider many algorithmic advances [3,5] and use stochastic (or deterministic) pulse trains to incrementally

\footnotetext{
${ }^{2}$ Consult the online documentation for a complete overview at https://aihwkit. readthedocs.io.
} 
perform the theoretical rank update

$$
w_{i j}^{(t+1)}=w_{i j}^{(t)}+\lambda d_{i} x_{j}
$$

where $d_{i}$ are the back-propagated error signals. One possible way to implement the update pass is to use stochastic pulse sequences of variable or fixed length, where the probability of having a pulse is proportional to the size of $x_{j}$ and $d_{i}$. In case of pulse coincidences, the weight element at crosspoint $i j$ is updated by a finite amount $\Delta w_{i j}$ (see [5]).

Importantly, due to physical constraints, the amount $\Delta w_{i j}$ can vary in a systematic way from crosspoint to crosspoint (device-to-device variations) or might have a systematic bias in one or the other direction (up versus down pulses). Moreover, the step $\Delta w_{i j}$ might depend in a non-linear way on the actual conductance of the crosspoint value, depending on the physical properties of the material used as resistive element.

We thus provide a flexible way to choose the correct update response and fit the update behavior to the material properties under investigation, such as the amount of device-to-device variations in various parameters and additional pulse-to-pulse variations. We also provide a number of presets calibrated on hardware data (e.g. for ReRAM [6] characteristics, see Fig. 3). Example response curves are shown in Fig. 3 ,

Note that our framework differs significantly from the update approach implemented by DNN +NeuroSim. For instance, DNN+NeuroSim uses native PyToRCH operators to compute the accumulated gradient, which in case of batch size larger than one or generally for convolutions will accumulate $p$ outer products in digital, and then only discretize the accumulated result to calculate the pulse number and add pulse variations. This means that the outerproduct is actually done in digital and not analog. This will result in a dramatic under-estimation of the update noise properties for a system that performs the rank-1 update in place in parallel in analog (as suggested by [5]). Thus, we reimplemented convolution and linear operators in our $\mathrm{C}++$ core to ensure that gradient accumulation happens using parallel update in analog memory even for batch sizes larger than 1 or in case of convolutions.

Moreover, our framework draws the stochastic pulse trains and applies the update pulses (in case of a coincidence) sequentially on the devices, which are then updated according to the device model pulse response curve. These pulsed updates are highly optimized for GPU compute, where we provide hundreds of custom CUDA kernel templates that are automatically tuned for each tile, thus selecting the fastest template on the fly. Consequently, the full analog training simulation often takes only 2-5x longer than the conventional floating-point training 3 .

\footnotetext{
${ }^{3}$ Depending on DNN and device setting, e.g. 60s per epoch for VGG-8/CIFAR10 with parallel pulsed update versus 15s with floating point on a V100 GPU
} 
A
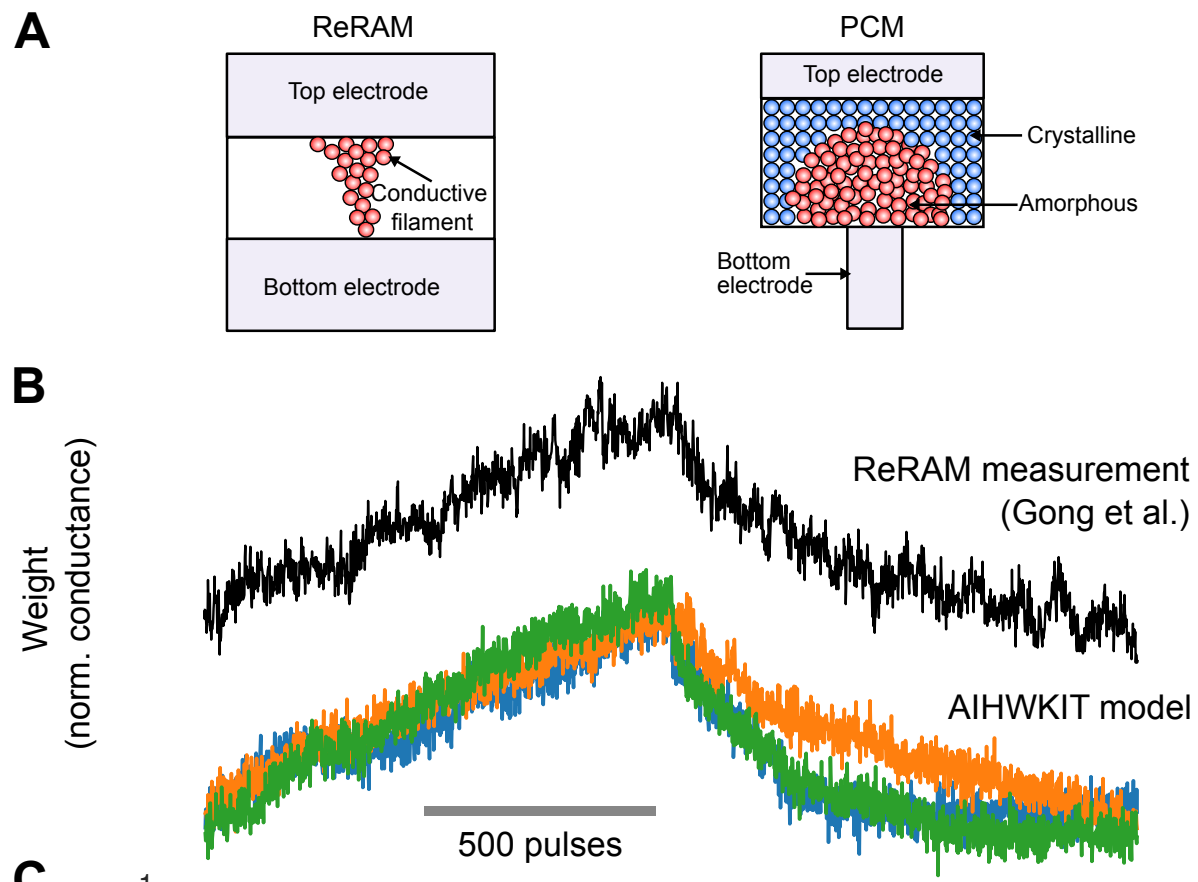

C

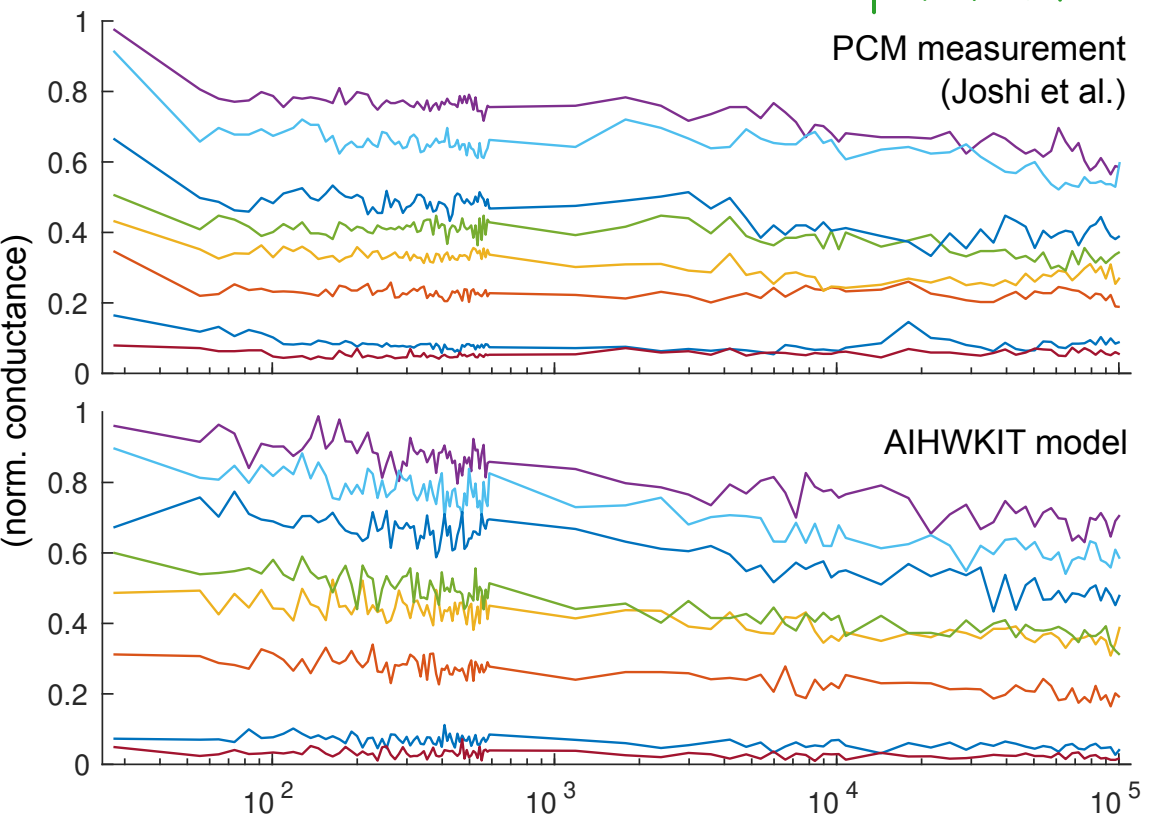

Time (s)

Figure 3: A) Schematic illustration of ReRAM and PCM devices. B) Pulse response of the experimental [6] and simulated ReRAM device (with device-todevice variations, write noise and, cycle-to-cycle variations). C) Experimentally obtained temporal evolution of PCM conductance [10] compared to that simulated by the statistical noise model of our inference tile. Note that we assume currently that all weights are programmed at the same time in the simulation, whereas in the experiment devices converge at different iterations of programming and there will be jitter in times at which drift starts in individual devices. This effect leads to the slight difference noticeable for small times (less than 1000 seconds) between model and experiment. 


\section{Advanced device models and optimizers}

All the tile parameters are chosen by setting the resistive device configuration. The default is having a single device per crosspoint. However, in many hardware designs, it might be advantageous to have multiple conductive elements within a "unit cell". Therefore, we provide a mechanism to define unit cells having an arbitrary number of devices (each potentially having different material properties), which can be updated one-by-one or all together. This allows defining two uni-directional devices per cell, a multi-device cell or a bi-directional single device cell with reference devices, by configuring the parameters of our analog tile accordingly.

Moreover, some analog optimization schemes have been proposed, where each analog tile of SGD is replaced with two analog tiles to create a coupled system by exchanging information between the two [3] tiles. We provide such constructs that can be configured to test different and new analog inspired optimization algorithms (see Fig. 4).

Lastly, a number of temporal noise processes are available, such as conductance decay, reset, and diffusion, which are all implemented with systematic device-to-device variability. These temporal processes are applied once per minibatch and are thus assumed to be negligibly small within the physical time a one mini-batch computation takes.

\section{$5 \quad$ Training for analog inference chips}

It has become increasingly apparent that accuracy on chips that are designed for inference benefit greatly from "hardware-aware" training, where the DNN workload is trained (or fine-tuned) in software with some analog noise and nonidealities in the forward pass, but with otherwise perfect backward and update pass [4,8, 10, 14]. Our framework supports hardware-aware training for inference. In particular, backward and update of a tile can simply be configured as being "perfect". Moreover, we provide weight noise functions for inference training, that reversibly add noise onto weights during a mini-batch (in forward and backward, but not during update pass) to train for noise resiliency.

Finally, we also provide a specific inference "tile" setting, which supports adding carefully calibrated, conductance-dependent programming noise, weight read noise and conductance drift onto a trained networks' analog weights during inference time and provides automatic global drift compensation (see eg. [10]). The noise strength and temporal profile is calibrated on a $1 \mathrm{M}$ phase-change memory (PCM) device array [10] (see Fig. 33).

Thus, any network can be tested for realistic inference accuracy and how the accuracy degrades over time. 


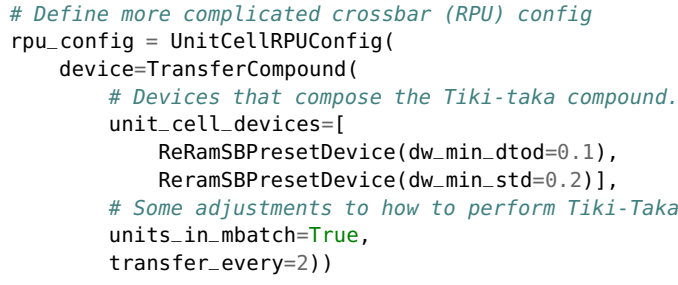

Figure 4: A more complex device configuration. This example implements the Tiki-taka modified SGD rule 3 for analog training. Once the rpu_config is defined, the DNN training is identical to Fig. 2,

\section{Extendability and open source contributions}

The reason of using PYTORCH as an AI framework is to have a system to flexibility define arbitrary DNN architectures. Our simulator inherits this flexibility in defining networks with both analog and digital components, where for the latter all features of PYTORCH can be used. Moreover, because of the modular structure of our code base, it is easy to extend and incorporate new features. For instance, the inference noise models use Python classes and could be easily derived to implement other drift characteristics.

Device update behavior, on the other hand, is internally computed in $\mathrm{C}++$. This core $\mathrm{C}++$ RPUCUDA library is designed for speed but retains a modular structure to make it also extendable. While a number of abstract device models are already provided, including a number of parameter presets, new device models can be implemented relatively easily by inheriting most properties of existing devices. In particular, if only the update response curve of a new material needs to be changed, many optimized kernel routines can be re-used, as only the update function itself needs to be written and added to the code (in $\mathrm{C}++$ for CPU and GPU).

In general, we follow an open source development model using GitHub standards, where we encourage contributions and discussions via an issue tracker, while using modern industry best-practices for software development (continuous integration, automated unit testing, code reviews) and packaging (semantic versioning, documentation, PEP adherence).

\section{Conclusion}

We present a new flexible open source software tool for simulating the functional aspects of analog AI with a clear features roadmap. It is solely focused on the algorithmic development and functional verification for ANN training and inference on emerging analog chips. This toolkit is not intended to estimate run time, latency, or power performance of a hardware chip. While this is 
very important in its own right, performance aspects are (largely) irrelevant to the functional characterization and need dedicated and complimentary tools. We hope that this toolkit will make it easier for researchers from AI hardware development and deep learning communities, to develop new algorithms and design new ANN topologies that are optimized for analog AI, hence experiencing the exciting new area of analog AI.

\section{References}

[1] G. W. Burr et al. Neuromorphic computing using non-volatile memory. Advances in Physics: X, 2(1):89-124, 2017.

[2] P.-Y. Chen et al. NeuroSim+: An integrated device-to-algorithm framework for benchmarking synaptic devices and array architectures. In 2017 IEEE International Electron Devices Meeting (IEDM), pages 6-1, 2017.

[3] T. Gokmen and W. Haensch. Algorithm for training neural networks on resistive device arrays. Frontiers in Neuroscience, 14, 2020.

[4] T. Gokmen, M. J. Rasch, and W. Haensch. The marriage of training and inference for scaled deep learning analog hardware. In 2019 IEEE International Electron Devices Meeting (IEDM), pages 22-3. IEEE, 2019.

[5] T. Gokmen and Y. Vlasov. Acceleration of deep neural network training with resistive cross-point devices: Design considerations. Frontiers in neuroscience, 10:333, 2016.

[6] N. Gong and other. Signal and noise extraction from analog memory elements for neuromorphic computing. Nature communications, 9(2102), 2018.

[7] W. Haensch, T. Gokmen, and R. Puri. The next generation of deep learning hardware: Analog computing. Proceedings of the IEEE, 107(1):108-122, 2018.

[8] S. Jain et al. Neural network accelerator design with resistive crossbars: Opportunities and challenges. IBM Journal of Research and Development, 63(6):10-1, 2019.

[9] S. Jain, A. Sengupta, K. Roy, and A. Raghunathan. RxNN: A framework for evaluating deep neural networks on resistive crossbars. IEEE Transactions on Computer-Aided Design of Integrated Circuits and Systems, 2020.

[10] V. Joshi et al. Accurate deep neural network inference using computational phase-change memory. Nature Communications, 11(2473), 2020.

[11] A. Paszke et al. Pytorch: An imperative style, high-performance deep learning library. 
[12] X. Peng, S. Huang, H. Jiang, A. Lu, and S. Yu. DNN+NeuroSim V2.0: An end-to-end benchmarking framework for compute-in-memory accelerators for on-chip training. arXiv:2003.06471, 2020.

[13] A. Sebastian, M. Le Gallo, R. Khaddam-Aljameh, and E. Eleftheriou. Memory devices and applications for in-memory computing. Nature Nanotechnology, 15:529-544, 2020.

[14] H. Tsai et al. Inference of long-short term memory networks at softwareequivalent accuracy using $2.5 \mathrm{M}$ analog phase change memory devices. In 2019 Symposium on VLSI Technology, pages T82-T83. IEEE, 2019. 